\title{
The Geo-Privacy Bonus of Popular Photo Enhancements
}

\author{
Jaeyoung Choi \\ Delft University of Technology \\ Netherlands \\ International Computer Science \\ Institute, USA \\ j.choi@tudelft.nl \\ Kevin Li \\ University of California, Berkeley \\ USA \\ kevinli7@berkeley.edu
}

\author{
Martha Larson \\ Radboud University \\ Delft University of Technology \\ Netherlands \\ mlarson@science.ru.nl
}

\author{
Gerald Friedland \\ Lawrence Livermore National \\ Laboratory \\ University of California, Berkeley \\ USA \\ fractor@eecs.berkeley.edu
}

\author{
Xinchao Li \\ Delft University of Technology \\ Netherlands \\ x.li-3@tudelft.nl
}

\begin{abstract}
Today's geo-location estimation approaches are able to infer the location of a target image using its visual content alone. These approaches typically exploit visual matching techniques, applied to a large collection of background images with known geo-locations. Users who are unaware that visual analysis and retrieval approaches can compromise their geo-privacy, unwittingly open themselves to risks of crime or other unintended consequences. This paper lays the groundwork for a new approach to geo-privacy of social images: Instead of requiring a change of user behavior, we start by investigating users' existing photo-sharing practices. We carry out a series of experiments using a large collection of social images to systematically analyze how photo editing practices impact the performance of geo-location estimation. We find that standard image enhancements, including filters and cropping, already serve as natural geo-privacy protectors. In our experiments, up to $19 \%$ of images whose location would otherwise be automatically predictable were unlocalizeable after enhancement. We conclude that it would be wrong to assume that geo-visual privacy is a lost cause in today's world of rapidly maturing machine learning. Instead, protecting users against the unwanted effects of pixel-based inference is a viable research field. A starting point is understanding the geo-privacy bonus of already established user behavior.
\end{abstract}

\section{KEYWORDS}

geo-privacy; geo-location estimation; usable privacy for multimedia retrieval

Permission to make digital or hard copies of all or part of this work for personal or classroom use is granted without fee provided that copies are not made or distributed for profit or commercial advantage and that copies bear this notice and the full citation on the first page. Copyrights for components of this work owned by others than the author(s) must be honored. Abstracting with credit is permitted. To copy otherwise, or republish, to post on servers or to redistribute to lists, requires prior specific permission and/or a fee. Request permissions from permissions@acm.org.

ICMR '17, June 6-9, 2017, Bucharest, Romania

(C) 2017 Copyright held by the owner/author(s). Publication rights licensed to ACM ACM ISBN 978-1-4503-4701-3/17/06 ..\$15.00

DOI: http://dx.doi.org/10.1145/3078971.3080543

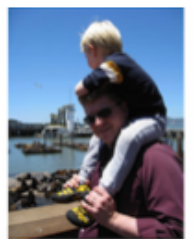

(a)

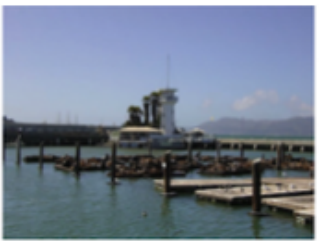

(b)

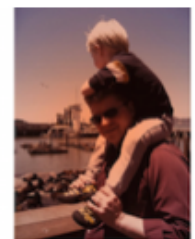

(c)
Figure 1: Geo-location Estimation can automatically infer the location of a target photo (a) shared online by a user if at least one visually matching, geo-tagged image, e.g., (b), a 'geo-propagator', exists in the background collection. However, widespread practices, such as enhancement filters (c) can serve to protect image geo-location information.

ACM Reference format:

Jaeyoung Choi, Martha Larson, Xinchao Li, Kevin Li, Gerald Friedland, and Alan Hanjalic. 2017. The Geo-Privacy Bonus of Popular Photo Enhancements. In Proceedings of ICMR '17, June 6-9, 2017, Bucharest, Romania, , 9 pages.

DOI: http://dx.doi.org/10.1145/3078971.3080543

\section{INTRODUCTION}

As multimedia retrieval technologies improve, it is possible in an increasing number of cases to automatically infer the location at which a photo was taken on the basis of its visual content. Most work on Geo-location Estimation (GLE), emphasizes the usefulness of geo-coordinates in organizing photo collections, and improving multimedia retrieval, e.g., [16, 30, 31]. However, the success of GLE in supporting users in browsing and finding photos depends critically on our ability as a multimedia research community to understand and mitigate its risks.

Users unknowingly release information about their location when they share photos, thus surrendering control of their own geo-privacy, and making themselves vulnerable. Relatively harmless vulnerabilities include the annoyance of unwelcome targeted advertisement. Worse risks also exist, for example, users can expose themselves to criminal attack. Even users who are cautious with 
publicly sharing photos, are vulnerable if their photos are passed from friend to friend, or if they are stored in unencrypted form, and the storage is hacked.

The key points about GLE for social images that are relevant for this paper are illustrated by the examples in Fig. 1. The leftmost image is a photo that has been uploaded by a user to a popular photo sharing platform (Flickr), and whose geo-location is correctly predicted by our GLE approach, but not predictable after a filter is applied (rightmost image). This geo-privacy 'bonus' of filters is a form of privacy by obfuscation [5] applied to the visual domain. The main approach we apply in this paper, which uses geometric visual matching, represents the state of the art in GLE, and is described in detail later. We also train a deep convolutional neural network inspired by [31] for comparison but, as we will see, the resolution of geo-estimation is much higher for the visual-matching-based approach.

We emphasize that we are not trying to compare different GLE methods and claim that our approach is superior, nor are we trying to argue that our proposed photo taking or enhancing behavior will provide complete geo-privacy protection against current and future GLE systems. Instead, the purpose of this paper is to lay the foundation for a new line of research directed at supporting users in protecting themselves, given their own natural photo-taking and sharing behavior.

At this point, we introduce the core mechanism of our approach. Retrieval-based GLE makes use of a large background collection containing photos for which the geo-location is already known, for example, because the photos are accompanied by GPS coordinates or manually geo-tagged by the uploading user. Here, we use 8.5 million images. The target image is used to retrieve 'geopropagators', visually matching images from the background collection. Geo-location prediction is accomplished by propagating the geo-coordinates of one or more geo-propagators to the target image.

In Fig. 1, the middle image is an example of a 'geo-propagator', one of the images used to infer the location of the target image (leftmost image). This image was taken by a different user, who either used a camera that recorded GPS coordinates, or provided the geo-location during upload. Note that retrieval-based GLE does not build a explicit model of a location, rather, a single photo uploaded by another user is sufficient to identify the geo-location at which the target photo was taken. In this example, the target photo and the geo-propagator clearly depict the same object/landmark. However, the visual match can also involve what could otherwise be considered unimportant background detail.

It is natural for the images (a) and (b) in Fig. 1 to be a cause for some alarm. The leftmost photograph was uploaded by a user, who most likely would assume that the location of the photo would be protected if he turned off the GPS of his cameras. As already mentioned, the unintended consequences range from annoying (e.g., for newly weds who were not planning to reveal their secret honeymoon destination) to downright dangerous (e.g., for a young mother targeted by a stalker). This alarm is the factor that triggered this paper. However, the basic insight that we build on is fundamentally a positive one: Users' photo taking and sharing behavior does not always lead to geo-privacy risks. If we can analyze natural behavior that protects privacy, then we take a step towards achieving our ultimate, long term goal, of developing multimedia technology that puts control over geo-privacy back into the hands of users.

The rightmost image (c) of Fig. 1 represents the widespread practice of filtering, which is naturally protective of geo-privacy. This image is comparable to image (a) from the user perspective. However, in contrast to (a), the location can not be correctly predicted via visual matching with images in the background collection. In the example, the "Toaster" Instagram filter has been applied. After application of the filter, the location of the image can no longer be correctly predicted by our GLE algorithm.

In short, this example points towards the existence of simple practices applied by users that naturally geo-cloak their images, i.e., prevent the locations of those images from being automatically predicted. The implication is that it is not necessary to introduce new, never-before-seen technologies in order to protect users' geoprivacy. Such technologies require user acceptance and widespread uptake before they can be effective. Rather, we can target new information retrieval technologies that build on behaviors that users naturally engage in, independently of privacy considerations. This paper represents a first step on the road to such technologies.

We take the position that GLE technology has both positive and negative implications for users. For this reason, we necessarily approach the topic of geo-privacy for social images, not with the goal of developing the ultimate privacy protecting algorithm, but rather, with the goal of attaining the correct balance between positive and unintended consequences. It is important to note that we are not asserting that every image must be geo-privacy protected/geocloaked. Indeed, there are many images taken and shared by users precisely for the reason of revealing geo-location, apparently to as many other people as possible. We do not propose that every picture of the Eiffel Tower or of the Taj Mahal should be enhanced to become visually distinctive. Rather, we strive to understand how users' behavior impacts geo-privacy because of its importance in situations where geo-location information is 'incidental' to images, and represents a potential, unintended geo-privacy leak. Because of the rate at which photos are taken and shared by users online the balance that we strive to achieve is necessarily a dynamic balance. A photo whose geo-location cannot be identified by our system today, might well be identifiable tomorrow, as more images are taken and added to the background collection. We address the dynamic balance by considering not only the geo-cloaking of individual images, but also the connection of how the ability of a given photo to compromise the geo-privacy of the user is related to the availability of other photos online.

The paper makes the following contributions:

- Danger of Geo-Location Estimation: Comparison of a stateof-the-art GLE system that uses image retrieval (visualbased matching) and a deep network-based classification approach.

- Image editing behavior: Experiments showing the way in which widespread image enhancements (e.g., filters and cropping) impact automatic GLE,

- Research challenges: Provide the motivation for a new line of research devoted to visual geo-privacy that protects 
users against unwanted affects of visual retrieval by leveraging their own natural behavior.

In the next section, we provide additional motivation for the paper and also cover related work. Then, we present the results of our experiment-based analysis. We finish with an outlook on the challenges to be tackled in the future.

\section{MOTIVATION AND RELATED WORK}

In this section, we take a closer look at the background and motivation for our work, and discuss its relationship to existing work.

\subsection{Image Privacy Classification}

Studies on privacy-aware image classification [27, 33] try to provide a safeguarding mechanism that relies on the automatic classification of privacy-related images by image sharing platforms. Such an approach attempts to alleviate the limitations of privacy settings in social networks. Although this approach may provide some measure of improvement in users' privacy, there is no guarantee that an online social network platform will incorporate necessary solutions to provide adequate protection to its users. For this reason, privacy solutions must focus on the users' side and factors directly under the users' control.

\subsection{The Importance of Geo-Privacy}

Privacy involves the ability to control information about oneself, and, by extension, we define geo-privacy as the ability to control information about one's location, both in the past and in the future. As mentioned in the introduction, leaks of personal geo-information can be annoying. The literature, however, tends to cover the more dangerous consequences, especially the phenomena of 'cybercasing', burglars automatically harvesting information about potential victims online [13]. cybercasing [13], generally, out-of-town homeowners. Ready-or-Not ${ }^{1}$ and PleaseRobMe ${ }^{2}$ serve to illustrate the privacy implciations of location disclosure in online social networks. Further, the phenomenon of stalking has been connected to opportunity [25], and, as such, geo-information in the hands of the perpetrator renders the victim more vulnerable.

In the real world, there are no failsafe protections against burglars and stalkers. Instead, people protect themselves by trading off convenience with conventional protection measures. Our goal in seeking to protect geo-privacy is to increase the number of measures at users' disposal to protect themselves.

There are obvious parallels between concerns about automatic face detection algorithms compromising privacy [29], and geolocation estimation algorithms compromising privacy. However, geo-location estimation is potentially more pernicious, since users are likely unaware of conditions under which a GLE algorithm can automatically predict the locations of their images. The first 'GLE condition' is the visual distinctiveness of the image content. The visual elements that allow GLE to geo-locate the image in the first column of Fig. 1 range from fairly obvious to barely noticeable. The second 'GLE condition' is the presence of 'geo-propagators' in the background collection that are visually closer to the target image than images taken at other locations. Although a user may

\footnotetext{
${ }^{1}$ app.teachingprivacy.org

2pleaserobme.com
}

have a rough idea of the number of photographs taken at a given location, there is no way to guess the existence of visually similar locations. The impossibility of deciding whether the GLE conditions are satisfied, mean that users are, an can be expected to remain, easily susceptible to inadvertently leaking geo-information when sharing photos.

Two points characterize the nature of the negative impact that automatic GLE of social images poses to user geo-privacy. First, GLE is dangerous not because it is able to identify the location at which an image is taken, but rather because it is able to do so automatically. Automatic processing is fast, and makes it possible to mine large collections of social images in search of vulnerabilities, as with cybercasing, mentioned above. Second, automatic processing can potentially identify location in cases that would be challenging for a human observer. The number of people who can identify the location of the images in Fig. 1 is relatively small. The location can, however, be identified by anyone with access to GLE technology. The goal in protecting geo-privacy is not to completely eradicate evidence of location in social images, but rather to slow down or hold back the ability of GLE algorithms to automatically process large quantities of social images and extract location information. Users should retain control over whether or not their images are geo-locatable or not.

\subsection{Social images and geo-location estimation}

This paper takes the position that it is important to understand how user behavior impacts automatic geo-location estimation technology, since this technology opens users to risks that they may be unaware of. It should be noted that this is a nuanced stance-we do not claim that all automatic geo-location estimation is bad, rather that we need to understand the extent to which users can control whether or not it can be applied to their photos. In this subsection, we briefly review the work on geo-location estimation. This work has generally emphasized the positive side of image GLE, and the potential it has to serve users in browsing and finding images.

Although this paper is devoted to protecting users against the unintended consequences of visual GLE, we point out that ultimately researchers need to address the unintended consequences of multimodal (such as visual/text or visual/audio) GLE.

2.3.1 Visual-based geo-location estimation. Visual-approaches to automatic geo-location estimation can be divided into two types of approaches. The first type, geo-constrained GLE approaches, attempts to predict the geo-location given a relatively restricted range of candidate locations. The second type, global-scale GLE approaches, attempt to predict the geo-location of photos any where on the surface of the earth. For this purpose, visual retrieval techniques applied to a large background collection of geo-tagged images are exploited, as mentioned in the introduction.

The first type of approaches, geo-constrained GLE, has included city-scale landmark recognition [7], estimation of location in target images using geo-informative visual attributes learned from training images of cities [18] [12], and classification [14].

Compared to the effort that has been devoted to geo-constrained GLE, there has been relatively less work dedicated to predicting locations at global scale. A major contribution in this direction was the approach by Hays and Efros [16]. They deployed various 
global visual representations to model the visual scene similarity between images and employed the Mean Shift Clustering approach to estimate the location. Further contributions were made by the Placing Task of the MediaEval multimedia evaluation benchmark, which addressed the challenge of location estimation for social images [15].

Here, we focus on a state-of-the-art GLE system called Distinctive Visual Element Matching approach, which was proposed by $\mathrm{Li}$ et al. [21]. Given a query image, a geo-location is predicted based on the evidence collected from images that have sufficient visual similarity to the query image and are also geographically close. The use of such 'geo-visual neighborhoods' to predict allows DVEM to improve over two major classes of previous visual-based retrieval approaches, addressing the disadvantages of both 1-NM and clustering [16].

Google's PlaNet [31] is a deep convolutional neural network $(\mathrm{CNN})$ approach that divides the surface of the globe into cells. The algorithm adapts cell size to the number of images in its training set that are labeled with geo-coordinates for a specific location location, i.e., a location that has more photos will be divided into finer cells. Each cell is considered a class, and is used to train a CNN classifier. Inspired by the result reported in [31], we trained a deep network and compared with the retrieval based approach by Li et al. [21].

2.3.2 Image augmentation for convolutional neural networks. A major factor in the recent success of deep convolutional neural networks has been the availability of vast quantities of data. Data augmentation, which effectively increases the amount of training data by imposing a variety of changes on the original data, has been shown to increase the classification accuracy of deep convolutional neural networks. The winners [32] of the 2016 ILSVRC scene classification challenge [26] used color augmentation, PCA jittering, random image interpolation, and crop sampling in their training. Upon first consideration, Instagram filters may appear to qualify as a perturbation that might improve the performance of GLE estimation if applied to the training data. However, our assumption is that the geo-privacy bonus effect arises because image enhancements constitute information loss, and for this reason we expect that adding filtered images to the training data will not deliver an improvement related to data augmentation, but rather will be detrimental to GLE performance.

\subsection{Weakness of other geo-privacy approaches}

Next we cover possible alternative approaches that could be taken to geo-privacy. We mention them here in order to highlight their weaknesses, and underline the importance of gaining insight into how users natural behavior can already serve to protect their privacy.

2.4.1 Image alteration for keypoint removal and re-injection. The forensics and image analysis communities have devoted quite a bit of effort to techniques that conceal images from SIFT-based image retrieval system by removing keypoints and forging in new keypoints $[9,10,17]$. These systems were studied in the context of copyright detection and explore the robustness of near duplicate detection systems. This work differs from our own in that social images uploaded by users are very diverse, and for this reason, the need to handle near-duplicates is not common in GLE. In [24], image obfuscation methods are proposed to protect privacy of users against threats caused by Internet bots that extract biometric and other features for data-linkage purposes.

There are two drawbacks to keypoint removal and re-injection technology for protecting user geo-privacy. First, these type of image alteration may add unwanted distortion and artifacts to the image. In contrast to our approach, such technology cannot take for granted that the aesthetics of the image will be preserved. Second, such technology requires users to add a separate step to their phototaking and image enhancement practices. A certain number of users would likely adopt the technologies, but large-scale adoption would demand an unprecedented level of acceptance.

2.4.2 Human-based solutions. So-called 'human-based solutions' would require users to change their behavior in response to awareness of the risks of online photo sharing. Social networking and photo-sharing websites are increasingly offering the opportunities to share photos privately. Researchers studying user behavior with respect to photo privacy have found that users experience a strong desire to participate in social sharing, which exerts a force on them to accept problems resulting from lack of control over their identity and disclosures [4]. The difficulty that users experience in dealing with complicated privacy settings is uncovered by [20] As a result of these difficulties, users make the choice of easy (i.e., public) sharing over privacy protective measures when they get overloaded. People are worried that the photo will not be shared properly and this impact their decision to share privately [1]. Computer scientists have developed many mechanisms to protect users' privacy, such as encrypted email and messaging. However, users are slow to adopt such solutions for a range of reasons. We reject solutions that require users to radically change their behavior for the simple practical reason that any approach to geo-privacy that is not actively used by users is effectively worthless.

2.4.3 Strength of simplicity. Recently, appreciation has arisen for simple, practical, filter-based approaches in service of privacy protection. The movement, typified by [6], has been led by researchers investigating how private information in images can be protected from human viewers. In contrast, in this work we investigate approaches to protect image content from algorithms.

However, like these researchers we recognize the value of simple approaches that are easy to apply in practice. Additionally, we point out the recent work that has been carried out on deceiving image classifiers that use deep neural networks [23]. In the context of privacy preservation, deceiving a classifier means protecting the content of an image. Here, we compare both classification and retrieval approaches to infer image content. Retrieval approaches may ultimately prove more difficult to defeat, since they require minimally one correct match, rather than a set of classifier training data.

\section{EXPERIMENTAL SETUP}

In this section, we set the stage for our analysis of the interaction of automatic GLE technology with user behavior by describing the setup of our experiments. 


\subsection{Data set and evaluation}

In order to ensure the validity and impact of our experimental results, we need a data set that is representative in scale for the state of the art in social image geo-location estimation. The data should also publicly be available, in order to make it possible for researchers who follow us to reproduce the experimental findings of this paper. We choose the MediaEval Placing Task 2013 Data Set $[15]^{3}$, a Creative Commons licensed set of images gathered from Flickr, which fulfills both of these criteria. Our experiments consist of two parts, GLE matching, and result analysis, and call for the application of both commercial tools and manual analysis. In order to ensure that we do not surpass the bonds of the resources at our disposal, we concentrate our experiments on a set of target images (also called, 'query images') that were taken in a specific region, San Francisco, California. We choose our region to be highly dense, since in this way it can be considered representative of the state which many regions of the world can be projected to reach, as the number of images taken by users continues to grow in future years. The total number of background collection images is 8,539,050 and the target images is 2,006 .

The results of our experiments are reported in terms of two different kinds of evaluation. First, the evaluation performance of the image GLE system. This is measured in terms of the percentage of photos whose geo-locations were correctly estimated with a given distance threshold (i.e., radius) of their ground truth location. The ground truth of the photos is the position at which the photographer was standing when the picture was taken. We use the geo-coordinates recorded by the GPS of the user's camera, or assigned by hand to the user during the process of uploading to Flickr. We focus on two distance thresholds: 100 meters and 1 kilometer, under the assumption these are relevant for privacy concerns. Second, we report the performance the results of our experiments in terms of percent cloaked. The percent cloaked is the proportion of the number of images that can be correctly geo-located with the original image, that can no longer be correctly geo-located once a specific 'enhancement', e.g., a filter, has been applied.

\subsection{Visual geo-location estimation approach}

Next we describe the GLE systems that were used for the experiments: DVEM, the search approach based on geometric visualfeature matching and a deep convolutional neural network based approach.

3.2.1 DVEM: Distinctive Visual Element Matching. Distinctive Visual Element Matching [21] is a search-based approach to the prediction of the geo-location of an image. This algorithm uses local image features (upright SIFT) for retrieval, and then creates geo-clusters in the list of image search results. It adopts the top ranked clusters and validates the visual match at the cluster level (rather than at the level of an individual image) using a geometric verification technique for object/scene matching. DVEM leverages the occurrence of visual elements that are discriminative for specific locations.

3.2.2 Deep Convolution Neural Network. As many neural network approaches are achieving the state of the art result in a variety

\footnotetext{
${ }^{3}$ http://www.st.ewi.tudelft.nl/ hauff/placingTask2013Data.html
}

of tasks, we also implemented a deep convolutional neural network for location estimation based on PlaNet [31]. The world map was partitioned into grids with respect to the density of the photos using Google's S2 library ${ }^{4}$ with $t_{1}=5000$ (maximum number of photos per grid) and $t_{2}=50$ (minimum number of photos per grid). We employed the GoogleNet [28] model with pre-trained weights from Places365 Dataset [34]. We trained our model by fine-tuning the fully connected layer and the last inception module using AdaGrad [11] with a learning rate of 0.2 to minimize cross-entropy loss with L2 regularization with a regularization constant of 0.01 . Training was stopped when training loss converged. Geo-location labels were naively assigned as the average latitude and longitude values of the training images in each grid.

\section{GEO-LOCATABLE SOCIAL IMAGES}

First, we discuss the overall performance of our image GLE systems. Figure 2 shows the performance of two visual geo-location estimation systems. DVEM is the visual-based matching (i.e., image retrieval) approach using upright-SIFT descriptors and DCNN represents the deep convolutional neural network approach. Each number represents the percentage of query images whose geolocations are correctly estimated within the distance thresholds from the groundtruth. We see that, DVEM system outperforms the DCNN system, and is able to correctly estimate the location of nearly $18 \%$ of the photos in the test collection.

Note that although this is the first time that DVEM and DCNN results have been reported for a problem at this scale, the focus here is not on the image GLE algorithm itself, but rather on widespread user photo-taking and enhancement practices. For this reason, it is important that the DVEM algorithm is the state of the art, but we are not looking to improve it, but rather we are investigating the factors that negatively impact its performance. When compared to the ultimate target of image GLE, to correctly geo-locate $100 \%$ of social images, our advanced system, which can only geo-locate $18 \%$ of images seems rather unthreatening. Unfortunately, this feeling is a false sense of security. Recall from above, that geo-privacy is compromised by large scale mining of images that searches for vulnerable victims to target. For people who leak visual geo-information by mistake, the potential of $18 \%$ to translates into direct damage is something that the community needs to take seriously, given that in $7.40 \%$ of the cases, the information is exact to the 100 meters level. The current state of GLE technology is a strong motivator to start, as is our goal in this paper, on the large mission of understanding how control over visual geo-information can be put in the hands of the user.

\section{IMPACT OF IMAGE ENHANCEMENT}

In this section, we examine practices of image enhancement that are in widespread use among users who share photos online. We investigate how enhancement affects automatic GLE of individual photos, and also how enhancement practices scale up as they are used not just by individual users, but by many users contributing images to the background collection.

\footnotetext{
${ }^{4}$ https://github.com/google/s2-geometry-library-java
} 


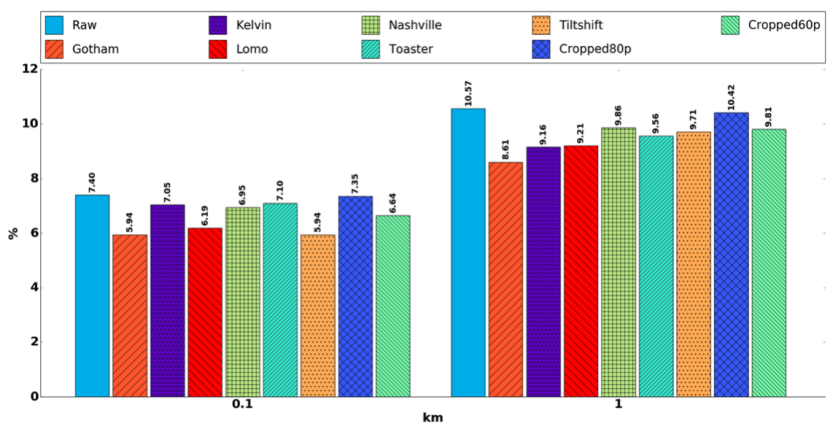

(a) DVEM

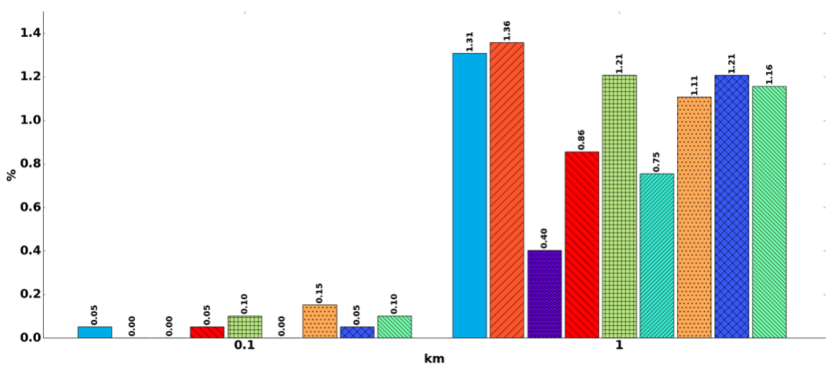

(b) DCNN

Figure 2: Two charts show the percentages of images correctly geo-located within an $100 \mathrm{~m}$ and $1 \mathrm{~km}$ radius from the groundtruth for the (a) DVEM and (b) DCNN approaches. Note that the accuracy (values of $y$-axis) of DCNN was much lower than DVEM and scale of $y$-axis is adjusted to show the effect of each photo enhancement.

\subsection{Users' behavior and image enhancement}

We define image enhancement as filters and other edits that people apply during or after image capture in order to heighten the attractiveness of the image. Specifically, we are interested in enhancements that are highly popular, since these best reflect widespread natural user behavior, which is the focus of our study. We focus on filters and features that are available to users on Instagram ${ }^{5}$, one of the most widely used photo sharing/social networking services.

Perhaps the simplest thing that users do to make their images more attractive is cropping. Cropping is often used to cut out clutters in the image and helps the viewer to concentrate on the main subject. Images lose some of its contents along the edge lines when cropped. We are interested in cropping because of its potential to cut out incidental matches in background that could allow photos to be geo-located. Next, we are interested in tilt-shift. Tilt-shift photography manipulates focus and depth-of-field to yield photos that have selective focus, often mimicking a miniature model. Instagram provides a post-production fake tilt-shift feature. Unlike cropping, tilt-shifted image still retains all of the original pixels, although the areas outside the focus point becomes blurred. We are interested in tilt-shift because it is popular, and also because the blur potentially eliminates matches in background.

\footnotetext{
${ }^{5}$ https://instagram.com/
}

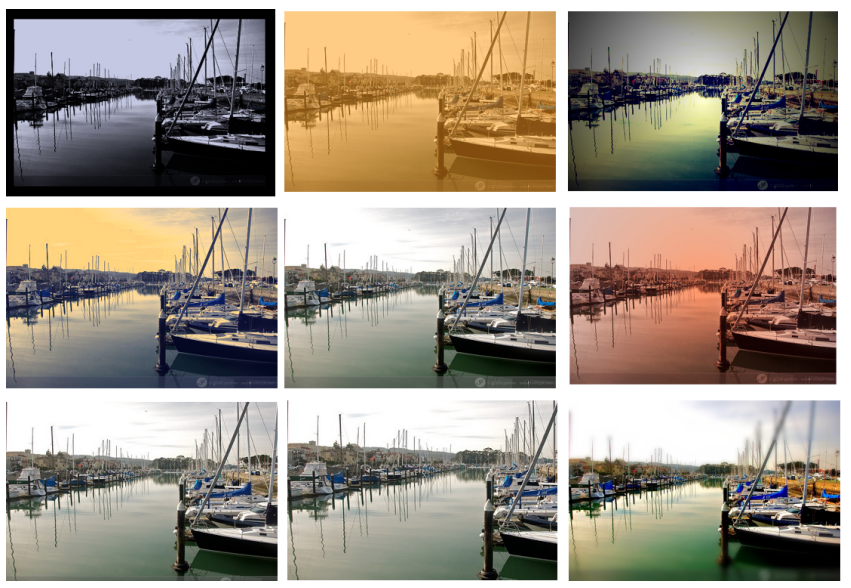

Figure 3: Image enhancements (popular on Instagram) use in our experiment. Center is the original image. Clockwise from top left: Gotham, Kelvin, Lomo, Toaster, Tiltshift, Crop40\%, Crop20\% and Nashville.

We focus a large number of experiments on filters. A filter is a preset recipe for adjusting various photographic settings (exposure, contrast, color balance, etc) of a photo to give it certain look and feel. Instagram offers a large number of filters that are carefully tailored, and provide users with a convenient means of enhancing the attractiveness of the photo, with no need for special skill [2]. We are interested in Instagram filters, since their popularity strongly suggests that users feel that they add value to images. Effectively, these filters represent a common practice already applied by users that potentially results in a loss of visual information in images, that can provide a geo-cloaking effect.

Instagram currently provides 40 filters for use. Among the popular filters are Kelvin, Lomo, Nashville, and Toaster. We have selected these filters for our study because they have a relatively strong influence on the original image, and for this reason provide a promising jumping-off point to study geo-cloaking effects. Although the Gotham filter has been discontinued by Instagram, we chose to also include it in our study, since effects are very dramatic with lots of contrast added. The following gives an overview of the filters. Figure 3 shows an example with each filters' effect.

- Gotham filter produces a black \& white, high contrast image with bluish undertones.

- Kelvin filter applies a strong peach/orange overlay.

- Lomo filter boosts contrast, and applies vignette (i.e., reduction in brightness/saturation around the edges).

- Nashville filter gives a washed out 80 s fashion photo feel with warmed up temperature, lowered contrast and increased exposure.

- Toaster filter features vivid colors with pink/orange glow out of the center.

To carry out the experiments we applied the enhancements to the target photos (i.e., test photos), and, for the final experiment, also to images in the background collection. Instagram filters were applied 
using ImageMagick ${ }^{6}$ in a Python wrapper. Cropping was done using Smart Cropping service provided by Imagga ${ }^{7}$. The Imagga cropping API analyzes images in terms of composition, color and object localization, and attempts to crop in a way that the main subject material of interest remains in the photo. Cropping was applied to crop out $20 \%$ and $40 \%$ of the original image, respectively. The aspect ratio of the photo was preserved. The tilt-shift effect was applied with a simple Python script [22]. We took the center point of a cropped image generated by Smart Cropping as the focus point of the original image, and the $y$-axis value of the center point was used to apply linear tilt-shift.

\subsection{Geo-cloaking effects of image enhancement}

Our experiments revealed that our enhancement filters demonstrated a clear ability to protect the location of images from being automatically predicted by GLE. An overview of the geo-cloaking performance of the filters is provided in Table 1.

\begin{tabular}{|c|c|c|c|c|c|c|c|c|}
\hline & \multicolumn{5}{|c|}{ Filter } & \multicolumn{2}{c|}{ Crop } & Tilt \\
\cline { 2 - 10 } & GOT & KEL & LOM & NAS & TOA & $\mathbf{2 0 \%}$ & $\mathbf{4 0 \%}$ & shift \\
\hline DVEM & 19.04 & 9.80 & 14.3 & 6.45 & 7.28 & 12.9 & 1.12 & 8.40 \\
\hline DCNN & 0 & 70.4 & 33.3 & 3.7 & 44.4 & 7.4 & 7.4 & 7.4 \\
\hline
\end{tabular}

Table 1: Net geo-cloaking percentage for DVEM and DCNN: Ratio between the number of images that can be automatically geo-located after and before an enhancement has been applied. (Filters: GOTham, KELvin, LOMo, NAShville, TOAster. Crop $20 \%$ and $40 \%$ represents the percent of the image cropped.) Higher number shows more geo-cloaking effect. Note that these values are relative gain from the images that were correctly geo-located.

These results are worth exploring further, since they clearly show that users' natural behavior can serve to help protect them from geoprivacy leaks, without requiring them to adopt new, yet-unknown technology or applications, or radical changes in their current photo sharing practices. For both DVEM and DCNN approaches, many filters do impressively well.

These findings suggest that geo-location-specific information is being naturally removed from the photo, as the user filters the photo to enhance its attractiveness. Examples of the protective effect of enhancements at work are provided in Fig. 4.

A major insight we can gain from this chart is the the ability of enhancements to geo-cloak photos is comparable at both the $100 \mathrm{~m}$ as well as the $1 \mathrm{~km}$ level, although cloaking is slightly more effective at $100 \mathrm{~m}$.

\subsection{Collective image enhancement patterns}

Our experiments so far have yielded a quite satisfying insight-we have determined that the use of image enhancement filters can lead to the substantial reduction of the ability of a geo-estimation system to correctly predict the location of an image. However, an obvious question to ask is whether enhancements might lose their

\footnotetext{
${ }^{6} \mathrm{http}: / /$ www.imagemagick.org

${ }^{7}$ https://imagga.com/technology/smart-cropping-and-slicing.html
}
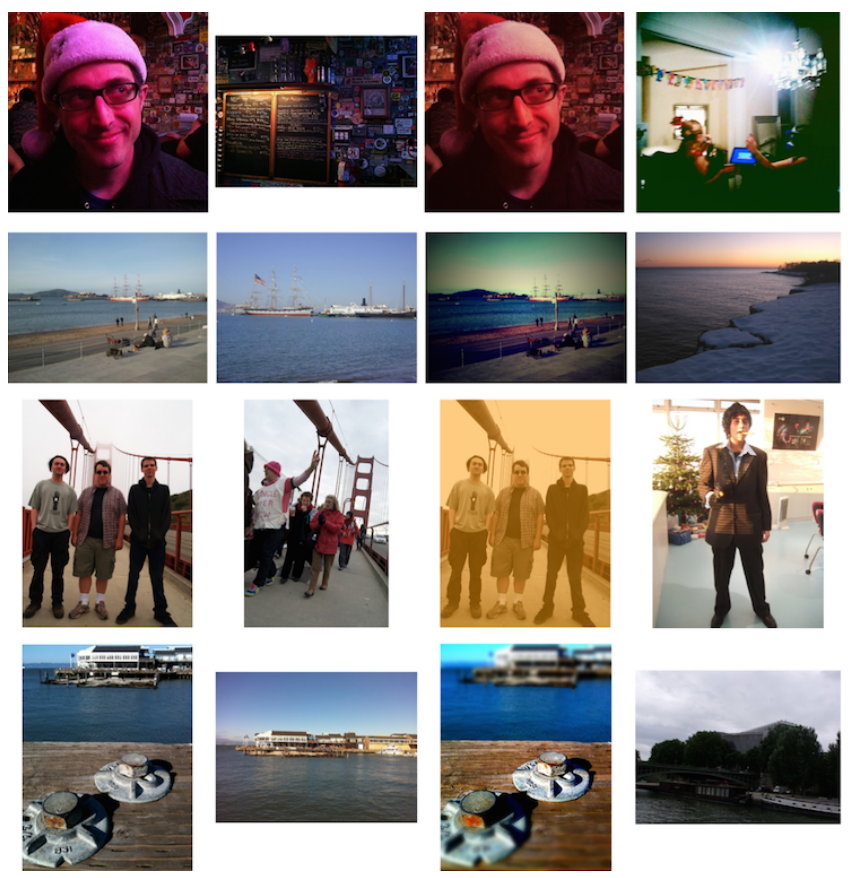

Figure 4: Example images whose locations are protected after enhancement. First column is the target image; second column is one of the 'geo-propagator' in the background collection; third column is the target protected with enhancement; fourth column is a close visual neighbor of the enhanced target (calculated with respect to a standard nearest neighbor visual matching in order to illustrate the effect). From top to bottom, the enhancements are: Toaster, Lomo, Kelvin, tilt-shift.

power to protect geo-privacy as the number of enhanced images in the background collection grows. Our final experiment takes a look at what happens to the ability of enhancements to block GLE location by the DCNN approach when training set images are themselves enhanced (original images augmented with enhanced images). In real life, there are two considerations that would impact how filtered images would accumulate in the set of images available creating the GLE system in a training set: first, the number of images that is filtered overall, and, second, the number of images that are filtered with a given type of filter. Here, we chose to focus on a scenario that constitutes a 'worse case' for geo-cloaking. In particular, we investigate the effects when the same enhancement is applied to both the target image and also to the training set images. We perform this experiment using the DCNN system and also report the result of a nearest neighbor based system [8] for comparison.

Table. 2 reports the percentages of enhanced target images that were geo-located when the background collection for nearest neighbor approach [8] consists of enhanced images and training set for DCNN includes both original and the enhanced images. Our hypothesis that filters introduce a loss of information for automatic GLE approaches is supported by the result shown here. 


\begin{tabular}{|c|c|c|c|}
\hline \multicolumn{2}{|c|}{} & BNN [8] & DCNN \\
\hline Original & $\mathbf{1 0 0 m}$ & 1.51 & 0 \\
\cline { 2 - 4 } Back. Collect. & $\mathbf{1 k m}$ & 4.48 & 0.25 \\
\hline Enhanced & $\mathbf{1 0 0 m}$ & 1.33 & 0 \\
\cline { 2 - 4 } Back. Collect. & $\mathbf{1 k m}$ & 4.08 & 0.1 \\
\hline
\end{tabular}

Table 2: Comparison of percentage of enhanced (Gotham filter) target images whose location is correctly predicted using the original collection, and using both the original collection and the images with enhancement. In both systems, including filtered images in the background collection (nearest neighbor approach [8]) or training set (DCNN) did not improve the performance.

Overall, the results of this experiment point to the conclusion that a growth in the use of filters will not reduce their ability to geo-cloak users' photos. In fact, enhancing the collection slightly reduces the ability of the GLE approach to correctly predict the location of images. With these results we are apparently observing that filters introduce a loss of that sort of information that is important in order for automatic GLE approaches to function optimally.

\section{VISUAL GEO-PRIVACY CHALLENGES}

We have argued in this paper that the current state of geo-location estimation technology warrants concern about the geo-privacy of users who take photos and share them online. The conditions under which the location of a photo can be automatically estimated depend both on the photo, and on the contents of the data available to use as the background collection or training set. Since these factors extend beyond what a user can be expected to be aware of, it is impossible for individual users to assess the risks to their own geo-privacy. Instead, they need support from technologies developed by multimedia information retrieval experts.

Our ultimate goal is to give users more control over geo-privacy by supporting them in preventing unintended leaks of geo-information while sharing photos online. Simple solutions, such as 'No-sharing', fail because of the immediacy of other concerns during the photo sharing process. Even if users share only privately, photos can fall into the wrong hands.

This paper has pointed out that although geo-privacy concerns are growing, the situation is not hopeless. Rather there are valuable insights that can be provided by research in the area of visual information retrieval. We have shown that users' own photo taking and image enhancement behavior actually has a contribution to make to protecting their privacy. We have systematically evaluated popular practices in image enhancement, and shown their impact on state-of-the-art geo-location estimation technology. Although certain Instagram filters are effective in helping to protect privacy, there is no silver bullet solution. Instead, more work is needed to understand the interactions of enhancements.

One part of the solution is obviously educating users, and a growing number of efforts are under way to actually teach users about social multimedia privacy [3]. However, an important part of the solution involves technical challenges.
Research dedicated, for example, to techniques that approximate the background collection, could take a step nearer to fitting the collection in memory, and thereby a step nearer to running a geoprivacy aware camera from a mobile phone. We envision such an application would also recommend to the user filters that add Instagram style enhancements, but that are specially chosen to protect geo-privacy. The key challenge of the geo-privacy aware camera is that it should fit seamlessly into currently widespread photographic practices, e.g., use of image enhancements.

Looking towards the future, there is much work to be done. The simplest, most pressing step to be taken is to avoid falling into the currently common language use, e.g., of [19], which refers to techniques that foil machine learning algorithms as 'attacks' independently of a well-specified use scenario. A neutral term such as 'cloaking' projects the positive importance of techniques, such as those that we have discussed in this paper, that inhibit automatic inference. Beyond geo-privacy there are numerous other application scenarios in which users might prefer to defeat machine learning inference to protect the information depicted in images that they share. If we are to create automatic inference systems using data that was generated by users, our first responsibility is to protect the interests of our users.

Moving forward in the longer term, it is critical that research focus both on improving performance of automatic geo-location estimation, but also on allowing users to protect themselves against it. The closer that geo-privacy protection can stay to people's own, already-existing, natural behavior, the greater the chance that it will be effective for the general user population in practice.

\section{ACKNOWLEDGMENTS}

Part of the experimentation for this work was carried out on the Dutch national e-infrastructure with the support of the SURF Foundation. It was also partially supported by a National Science Foundation Grant No. CNS 1514509 and a collaborative LDRD led by Lawrence Livermore National Laboratory (U.S. Dept. of Energy contract DE-AC52-07NA27344). Any findings and conclusions are the authors', and do not necessarily reflect the views of the funders.

\section{REFERENCES}

[1] Shane Ahern, Dean Eckles, Nathaniel S Good, Simon King, Mor Naaman, and Rahul Nair. 2007. Over-exposed?: privacy patterns and considerations in online and mobile photo sharing. In Proceedings of the SIGCHI conference on Human factors in computing systems. ACM, 357-366.

[2] Saeideh Bakhshi, David A Shamma, Lyndon Kennedy, and Eric Gilbert. 2015. Why We Filter Our Photos and How It Impacts Engagement. In Proceedings of International Conference on Web and Social Media. 12-21.

[3] Julia Bernd, Blanca Gordo, Jaeyoung Choi, Bryan Morgan, Nicholas Henderson, Serge Egelman, Daniel D Garcia, and Gerald Friedland. 2015. Teaching Privacy: Multimedia Making a Difference. IEEE MultiMedia 22, 1 (2015), 12-19.

[4] Andrew Besmer and Heather Richter Lipford. 2010. Moving beyond untagging: photo privacy in a tagged world. In Proceedings of the SIGCHI Conference on Human Factors in Computing Systems. ACM, 1563-1572.

[5] Finn Brunton and Helen Nissenbaum. 2015. Obfuscation: A user's guide for privacy and protest. MIT Press.

[6] Serdar Çiftçi, Pavel Korshunov, Ahmet Akyüz Oğuz, and Touradj Ebrahimi. 2015. Using False Colors to Protect Visual Privacy of Sensitive Content. In SPIE Human Vision and Electronic Imaging. 93941L-1-93941L-13.

[7] David M Chen, Georges Baatz, Kevin Köser, Sam S Tsai, Ramakrishna Vedantham, Timo Pylvänäinen, Kimmo Roimela, Xin Chen, Jeff Bach, Marc Pollefeys, and others. 2011. City-scale landmark identification on mobile devices. In Proceedings of the IEEE Conference on Computer Vision and Pattern Recognition. IEEE, 737-744.

[8] Jaeyoung Choi, Martha Larson, Xinchao Li, Gerald Friedland, and Alan Hanjalic. 2016. Where to be wary: The impact of widespread photo-taking and image 
enhancement practices on users' geo-privacy. arXiv preprint arXiv:1603.01335 (2016).

[9] Andrea Costanzo, Irene Amerini, Roberto Caldelli, and Mauro Barni. 2014. Forensic Analysis of SIFT Keypoint Removal and Injection. IEEE Transactions on Information Forensics and Security 9, 9 (July 2014), 1450-1464

[10] Thanh-Toan Do, Ewa Kijak, Teddy Furon, and Laurent Amsaleg. 2010. Deluding Image Recognition in SIFT-based CBIR Systems. In Proceedings of the ACM Workshop on Multimedia in Forensics, Security and Intelligence (MiFor '10). 7-12.

[11] Hazan E. Singer Y. Duchi, J. 2011. Adaptive Subgradient Methods for Online Learning and Stochastic Optimization. FMLR 12 (2011), 2121-2159.

[12] Quan Fang, Jitao Sang, and Changsheng Xu. 2013. GIANT: Geo-informative Attributes for Location Recognition and Exploration. In Proceedings of the ACM International Conference on Multimedia (MM '13). 13-22.

[13] Gerald Friedland and Robin Sommer. 2010. Cybercasing the Joint: On the Privacy Implications of Geo-tagging. In Proceedings of the 5th USENIX Conference on Hot Topics in Security (HotSec'10). 1-8.

[14] Petr Gronat, Guillaume Obozinski, Josef Sivic, and Tomas Pajdla. 2013. Learning and calibrating per-location classifiers for visual place recognition. In Proceedings of the IEEE Conference on Computer Vision and Pattern Recognition. 907-914.

[15] Claudia Hauff, Bart Thomee, and Michele Trevisiol. 2013. Working Notes for the Placing Task at MediaEval 2013. In Working Notes Proceedings of the MediaEval 2013 Workshop.

[16] James Hays and Alexei A Efros. 2008. IM2GPS: estimating geographic information from a single image. In Proceedigns of the IEEE Conference on Computer Vision and Pattern Recognition. IEEE, 1-8.

[17] Chao-Yung Hsu, Chun-Shien Lu, and Soo-Chang Pei. 2010. Secure and robust sift with resistance to chosen-plaintext attack. In IEEE International Conference on Image Processing. 997-1000.

[18] Yannis Kalantidis, Giorgos Tolias, Yannis Avrithis, Marios Phinikettos, Evaggelos Spyrou, Phivos Mylonas, and Stefanos Kollias. 2011. VIRaL: Visual Image Retrieval and Localization. Multimedia Tools and Applications 51, 2 (Jan. 2011), 555-592.

[19] Erica Klarreich. 2016. Learning Securely. Commun. ACM 59, 11 (Oct. 2016), 12-14.

[20] Bart P. Knijnenburg, Alfred Kobsa, and Hongxia Jin. 2013. Preference-based Location Sharing: Are More Privacy Options Really Better? In Proceedings of the ACM SIGCHI Conference on Human Factors in Computing Systems (CHI '13). 2667-2676.

[21] Xinchao Li, Martha A Larson, and Alan Hanjalic. 2016. Geo-distinctive Visual Element Matching for Location Estimation of Images. arXiv preprint arXiv:1601.07884 (2016).

[22] matveyco. 2013. python-tilt-shift. https://github.com/matveyco/python-tilt-shift. (2013)
[23] Anh Nguyen, Jason Yosinski, and Jeff Clune. 2015. Deep Neural Networks Are Easily Fooled: High Confidence Predictions for Unrecognizable Images. In Proceedings of the IEEE International Conference on Computer Vision and Pattern Recognition.

[24] Andreas Poller, Martin Steinebach, and Huajian Liu. 2012. Robust Image Obfuscation for Privacy Protection in Web 2.0 Applications. In IS\&T/SPIE Electronic Imaging. International Society for Optics and Photonics, 830304-830304.

[25] Bradford W. Reyns, Billy Henson, and Bonnie S. Fisher. 2012. Stalking in the Twilight Zone: Extent of Cyberstalking Victimization and Offending Among College Students. Deviant Behavior 31, 1 (2012), 1-25.

[26] Olga Russakovsky, Jia Deng, Hao Su, Jonathan Krause, Sanjeev Satheesh, Sean Ma, Zhiheng Huang, Andrej Karpathy, Aditya Khosla, Michael Bernstein, Alexander C. Berg, and Li Fei-Fei. 2015. ImageNet Large Scale Visual Recognition Challenge. International fournal of Computer Vision (IfCV) 115, 3 (2015), 211252

[27] Eleftherios Spyromitros-Xioufis, Symeon Papadopoulos, Adrian Popescu, and Yiannis Kompatsiaris. 2016. Personalized privacy-aware image classification. In Proceedings of the ACM International Conference on Multimedia Retrieval. ACM, 71-78.

[28] Christian Szegedy, Wei Liu, Yangqing Jia, Pierre Sermanet, Scott Reed, Dragomir Anguelov, Dumitru Erhan, Vincent Vanhoucke, and Andrew Rabinovich. 2015. Going Deeper with Convolutions. In Proceedings of the IEEE Conference on Computer Vision and Pattern Recognition. 1-9.

[29] Yaniv Taigman, Ming Yang, Marc'Aurelio Ranzato, and Lars Wolf. 2014. Deepface: Closing the gap to human-level performance in face verification. In Proceedings of the IEEE Conference on Computer Vision and Pattern Recognition. IEEE, 1701-1708.

[30] Michele Trevisiol, Hervé Jégou, Jonathan Delhumeau, and Guillaume Gravier. 2013. Retrieving geo-location of videos with a divide \& conquer hierarchical multimodal approach. In Proceedings of the ACM International Conference on Multimedia Retrieval. ACM, 1-8.

[31] Tobias Weyand, Ilya Kostrikov, and James Philbin. 2016. PlaNet - Photo Geolocation with Convolutional Neural Networks. In Proceedings of the European Conference on Computer Vision (ECCV).

[32] Shicai Yang. 2016. Towards Good Practices for Recognition and Detection. (2016). http://image-net.org/challenges/talks/2016/Hikvision_at_ImageNet_2016.pdf.

[33] Sergej Zerr, Stefan Siersdorfer, and Jonathon Hare. 2012. Picalert!: A System for Privacy-aware Image Classification and Retrieval. In Proceedings of the ACM International Conference on Information and Knowledge Management. ACM, 27102712 .

[34] Bolei Zhou, Aditya Khosla, Agata Lapedriza, Antonio Torralba, and Aude Oliva. 2016. Places: An Image Database for Deep Scene Understanding. arXiv preprint arXiv:1610.02055 (2016). 